\title{
Immunology of the gut and oral tolerance
}

\author{
Umid Kumar Shrestha*
}

\section{DOI Name}

http://dx.doi.org/10.3126/jaim.v4i1.14176

\section{Keywords}

Gut, oral tolerance, commensal gut flora, immune privilege collapse, inflammatory bowel disease.

\section{Citation}

Umid Kumar Shrestha. Immunology of the gut and oral tolerance. Journal of Advances in Internal Medicine 2015;04(01):16-24.

\begin{abstract}
The pathogens and harmless antigens from the bacterial flora and food constantly expose the mucosal surface of the gastrointestinal tract. The mucosal epithelial cells act not only as a physical barrier, but also as a local immune system, which plays a vital role in defense and self-tolerance. The gut mucosal immune system comprises several compartments: Peyer's patches and lymphoid follicles in the colonic mucosa, and lymphocytes in the lamina propria and intraepithelial lymphocytes. Peyer's patches mediate antigen uptake via specialized epithelial cells ( $M$ cells) and are rich in B cells for class switching into IgA-secreting cells. IgA secretion is one of the primary defenses against pathogens at mucosal surfaces. The lamina propria contains a high proportion of activated and memory $T$ cells that allows rapid immune response against pathogens. In the physiological situation, mucosally encountered antigens induce tolerance of lamina propria and intraepithelial lymphocytes by modified antigen presentation, antigen-induced anergy, or deletion of $\mathrm{T}$ cells, or regulation of effector $\mathrm{T}$ cells by regulatory or suppressor $\mathrm{T}$ cells. Costimulatory molecules mediate cellular interaction and induce regulatory cytokines. While the absence of gut immune privilege to food results in food allergy, the consequences of immune privilege collapse to commensal gut flora is Inflammatory Bowel Disease (IBD). Hence, the knowledge of the homeostatic regulation of the intestinal immune system paves the way for the development of the new immunomodulatory drugs in the therapy of IBD. Moreover, the generation of immune mediated cells through orally fed antigens could be the area of research in the treatment of certain autoimmune diseases.
\end{abstract}

\section{INTRODUCTION}

The gastrointestinal tract has developed a unique immunological system because of its close proximity to the intestinal contents. The system has evolved in such a way that it does not respond to the presence of beneficial microbes, but retains the ability to react quickly to the presence of harmful organisms. The challenge faced by the mucosal immune system is to generate an immune response of appropriate magnitude to control the microbial invasion while maintaining the integrity of the epithelial barrier. An imbalanced or improper immune response can result in a variety of inflammatory disorders of the gut.

In normal conditions human gut mucosa is infiltrated with a large number of mononuclear cells due to continuous stimulation by luminal antigens. This state of "physiological" inflammation is tightly controlled, as several mucosal cells interact to maintain an appropriate local immune response. Moreover, gut-associated lymphoid tissue (GALT) must constantly distinguish harmless antigens that are present in food and on commensal bacteria from pathogenic microbes.

For better understanding of the mucosal immune response it is useful to divide the gut-associated lymphoid tissue into inductive and effector sites, although recent data indicate that these distinctions are not absolute.

\footnotetext{
* Corresponding author

Umid Kumar Shrestha, MD, PhD

Department of Medicine,

Manipal College of Medical Sciences, Pokhara, Nepal

E-mail: umidshrestha@gmail.com
} 


\section{The inductive site for the mucosal immune response}

The primary inductive sites are organized lymphoid aggregates placed in the wall of the small and large intestine. In the small bowel these aggregates are called Peyer's patches ${ }^{1}$ and are composed of specialized follicle-associated epithelium, a subepithelial dome overlying $B$ cell follicles that contain germinal centers, and interfollicular regions with high endothelial venules and efferent lymphatic vessels. Isolated lymphoid follicles are also present in high numbers in the wall of the large bowel and the appendix. Following ingestion, antigens and micro-organisms are transported from the gut lumen via specialized $M$ cells, which are scattered among conventional epithelial cells overlying the dome of Peyer's patch follicles. Compared with epithelial cells, with which they share a common progenitor cell, they have a pronounced capacity to transport a wide variety of substances and microbes to the subepithelial dome region. Here dendritic cells are the main antigen-presenting cells that bind bacterial products with their Toll-like receptors (TLRs). The latter are a part of the innate immune defense, recognizing conserved patterns on micro-organisms. Signals initiated by the interaction of Toll-like receptors with specific microbial patterns direct the subsequent inflammatory response. ${ }^{2}$ In the mouse model it has been shown that dendritic cells in different states of maturation exist at different sites of the Peyer's patches. Dendritic cells in the subepithelial dome process antigen as relative immature cells and then migrate to the $\mathrm{T}$ cell region and present antigen to naive T cells. Here they have the properties of mature dendritic cells, with high surface expression of costimulatory molecules such as CD40, CD80, and CD86 and adhesion molecules such as CD44 and intercellular adhesion molecule (ICAM)- $1 .^{3}$ It is suggestive that the intestinal $T$ cells with distinct regulatory abilities, such as those producing transforming growth factor (TGF) b and interleukin (IL)10, are stimulated by interaction with less differentiated dendritic cells in the Peyer's patches. ${ }^{3}$ In the follicle such $\mathrm{T}$ cells provide help for the B cell switch to $\operatorname{IgA}$, a process that is completed in the germinal center. IgA is the major mucosal immunoglobulin and can help to dispose of antigens without provoking the complement cascade. Following IgA switch and affinity maturation B cells migrate from the Peyer's patches to the mesenteric lymph node via efferent lymphatic vessels and finally to the lamina propria where they undergo terminal differentiation into plasma cells.

The question arises about the signals that make a B cell home to the gut and specifically produce IgA. It has been showed that the vitamin A metabolite retinoic acid produced by GALTderived dendritic cells confers gut tropism and, together with IL-6 or IL-5, induces IgA secretion. GALT-derived dendritic cells directly induce gut tropism in a retinoic-acid-dependent manner, and they induce class switching to IgA in B cells through a synergistic mechanism that also involves retinoic acid. This study is of clinical relevance as it might explain why vitamin A deficiency exacerbates diarrhoeal disease in malnourished children. ${ }^{4}$

The effector site for the mucosal immune response:

The main effector site of intestinal immune responses is the lamina propria, where mature $T$ and $B$ cells migrate following induction in the Peyer's patches. The lamina propria contains a large and heterogeneous group of lymphoid and myeloid cells; in addition to lymphocytes, there are macrophages, dendritic cells, neutrophils, and mast cells. Effector mechanisms that protect mucosal surfaces include cytotoxic $T$ cells and effector $\mathrm{CD}^{+} \mathrm{T}$ cells for cytokine production and IgA response. Lamina propria $\mathrm{T}$ cells are mainly $\mathrm{CD}^{+} \mathrm{T}$ cells $(60-70 \%)$, the majority of which express the abT cell receptor (TCR; 95\%) just as in peripheral blood. ${ }^{5}$ However, lamina propria $\mathrm{T}$ cells differ from peripheral blood lymphocytes in that they are in a more highly activated state and express CD25 and HLA-DR antigen, presumably as a result of continuous exposure to luminale antigens, but have a decreased proliferation rate when stimulated by mitogen or specific antigens. ${ }^{6}$ Another characteristic of lamina propria T cells is that they produce high levels of cytokines when stimulated with mitogen or via CD2.7-9 Lamina propria $T$ cells have a mature or memory phenotype, indicated by the surface markers CD44 ${ }^{\text {high }}, \mathrm{CD} 62^{\text {low }}, \mathrm{CD} 45 \mathrm{RB} \mathrm{B}^{\text {low }}$ (mice)/CD45RO ${ }^{+}$(human) and high levels of the integrin $\mathrm{a}_{4} \mathrm{~b}_{7} \cdot{ }^{10-12}$ Taken together, lamina propria $\mathrm{CD} 4^{+} \mathrm{T}$ cells are highly differentiated effector cells with a raised threshold of activation that prevents immune responses to harmless intraluminale antigens. $\mathrm{CD} 8^{+} \mathrm{T}$ cells account for about $30-40 \%$ of $\mathrm{T}$ cells in the lamina propria. This cell population contains cytolytic effector cells, which seem to control the level of viral infection and other micro-organisms that share an intracellular stage in their life cycle in the lamina propria. ${ }^{13} \mathrm{~A}$ more restricted $\mathrm{T}$ cell population develops independently of the Peyer's patches and is integrated in the epithelial layer. These intraepithelial lymphocytes (IEL) are predominantly $\mathrm{CD} 8^{+} \mathrm{T}$ cells. In humans 5-30\% of small intestinal IELs bear a gdTCR and lack CD4 and CD8 or are $\mathrm{CD}^{+} .^{14,15}$ Predominant CD8 expression implies that IELS react in a $\mathrm{MHC}$ I restricted manner to antigens. However, the composition of IEL is different according to the anatomical sites. While most human jejunal IEL are $\mathrm{CD} 8^{+}$, there are much more CD4- CD8- IEL in the ileum and large bowel, most probably due to the different intraluminale content of microflora and food. ${ }^{15}$ The CD8 molecule on IEL is expressed predominantly as a homodimer of the CD8a-chain. The phenotypic complexity of IEL therefore suggests that they have different functions. It has been demonstrated that $\mathrm{CD}^{+} / \mathrm{CD} 8$ and the $\mathrm{CD} 4 / \mathrm{CD} 8$ 
double-positive IEL subset support the differentiation of Peyer's patch B cells into immunoglobulin producing cells. Furthermore, surface expression of $a_{4} b_{7}$ integrin, the ligand of which is E-cadherin on epithelial cells, ${ }^{16}$ and the production of keratinocyte growth factor by IEL ${ }^{17}$ suggest that some IEL populations play a role in maintenance of epithelial barrier integrity. In addition, TCRgdT cells may be involved in regulating the intestinal immune response. ${ }^{18}$ In the lamina propria IgA antibodies secreted by plasma cells keep the homeostasis of the epithelium by interacting with antigen within the gut lumen after epithelial transcytosis or inside infected epithelial cells or by formation of immune complexes with antigen in the lamina propria. However, it is clear that the antibody response is highly dependent on T cell help. ${ }^{19}$ This cell network in the mucosal immune system is highly integrated by the expression of specific costimulatory and adhesion molecules and the production of effector molecules such as cytokines.

Immune privilege in the gut:

The intestinal immune system constantly encounters foreign antigen, whether it be dietary antigen, components of the commensal gut flora, or antigens associated with pathogenic microbes. The gut falls squarely among those organs that can be considered immune privileged. There are two integral branches to immune privilege in the gut: oral tolerance and tolerance to commensal gut organisms. ${ }^{20}$

\section{Oral tolerance}

Oral tolerance is defined as the induction of a state of systemic immune nonresponsiveness to orally administered antigen upon subsequent antigen challenge. This mechanism presumably prevents the development of an immune reaction or allergy against intestinal intraluminale antigens.

Most of data on oral tolerance have so far been obtained in the animal model of ovalbumin-TCR transgenic mice. T cells appear to be the major target of tolerance, and the reduction in antibody responses after antigen feeding are due to the reductions in helper activity rather than to a tolerization of $B$ cells directly. The major mechanisms of tolerance induction are clonal deletion, clonal anergy, and the induction of suppressor cells. ${ }^{21}$ IL-12, a Th1-directing cytokine, may be a key regulatory cytokine for these various pathways in the mucosal immune response. Factors that suppress IL-12 production by antigen-presenting cells result in suppressor or regulatory $T$ cells (Tregs) producing TGF-b and possibly IL-4 and IL-10, while factors that induce IL-12 production result in T cells producing the proinflammatory cytokine interferon (IFN) g. ${ }^{22-24}$ The nature and localization of the antigen-presenting cells responsible for tolerogenic presentation of fed antigens are unclear. $T$ cell activation and/or deletion can be rapidly observed in the
Peyer's patches of antigen fed mice. ${ }^{25}$

Dendritic cells, which are the main antigen-presenting cells in the Peyer's patches, are usually considered to have a high ability to activate $T$ cells constitutively. However, there is increasing evidence that targeting antigen to resting dendritic cells, which lack costimulatory molecules, favors the induction of tolerance rather than immunity. ${ }^{26,27}$ In addition, enterocytes, which express Major Histocompatibility Complex class II molecules constitutively but lack costimulatory molecules such as ICAM-1, 28,29 could also account for tolerogenic antigen presentation. Recent data indicate that Peyer's patches alone are not important for the induction of oral tolerance. In a mouse model with targeted mutation in the gene of the tumor necrosis factor (TNF) a family the inhibition of the development of Peyer's patches and mesenteric lymph nodes is associated with a loss of orally but not intraperitoneally induced peripheral immune tolerance. ${ }^{30}$

Commensal flora signaling and oral tolerance induction

As mentioned above, TLR signaling helps to communicate commensal microflora with the intestinal immune system and promote mucosal tolerance. Twelve TLR family members have been identified in mammals. ${ }^{31}$ The bacteria-responsive TLRs, TLR2, TLR4, TLR5, and TLR9, are among the most well studied TLRs; their ligands include bacterial lipoprotein/lipoteichoic acids, lipopolysaccharide (LPS), flagellin, and unmethylated bacterial and viral CPG DNA motifs, respectively. ${ }^{32}$ TLRs are expressed on a broad range of epithelial, endothelial, stromal, and hematopoietic cells, including immune cells such as macrophages, DCs, B cells, and some T cells. ${ }^{31,32}$ TLRs possess the so-called Toll/IL-1R homology (TIR) cytoplasmic signaling domains. When a microbial component engages a TLR, it dimerizes and recruits TIR-domain-containing adapter molecules to its TIR domain. These molecules include myeloid differentiation factor 88 (MyD88), TIR-domain-containing adapter protein/MyD88-adapter-like, TIR-domain-containing adapter-inducing interferon-b (TRIF), and TRIF-related adapter molecule. ${ }^{31}$ The specificity and diversity of cytokine production and other effector functions downstream of TLR signaling result largely from differential recruitment of these adapter molecules. ${ }^{33-35}$ For example, TLR4 has been shown to activate nuclear factor-kB (NF-kB) through the common MyD88 signaling pathway shared by all the TLRs except TLR3; however, TLR4 also uses a MyD88-independent TRIF signaling pathway, which has been shown to be critical for inducing costimulatory molecule expression in DCs and for optimal production at the messenger RNA level of the cytokines IL$12, \mathrm{IL}-18$, and IL-23. ${ }^{35}$ Many functions have been ascribed to TLR engagement in different cell types. TLR ligand binding has been shown to influence trafficking of intestinal lamina propria 
dendritic cells, ${ }^{36}$ to promote the generation of T-dependent, antigen-specific antibody responses by $\mathrm{B}$ cells, ${ }^{37}$ to moderate the expansion and suppressive function of Tregs, ${ }^{38,39}$ and to modulate the ability of DCs to display phagocytosed cargo in the context of major histocompatibility complex class II molecules. ${ }^{40}$ Since commensal microbes and pathogens possess complex combinations and subtypes of various TLR ligands, ${ }^{32}$ perhaps the combination of TLR signals induced by a particular microbe informs the immune cell whether a microbe is benign or not.

Microbes that colonize intestinal mucus have metabolic and transcriptional profiles that differ from their biofilmindependent counterparts. ${ }^{41,42}$ Perhaps this altered profile facilitates host/microbial cross talk at the intestinal epithelial surface, promoting host tolerance to these microbes. Individual commensal gut microbes have been shown to reduce the production of proinflammatory cytokines by intestinal epithelial cells (IECS) by modulating the location and activity of enterocytic transcription factors such as NF-kB. ${ }^{43-45}$ As the largest body surface to interact with the external environment, the intestinal epithelium (comprising IECs with tight junctions) serves as a barrier to unregulated transport and systemic dissemination of commensal and pathogenic microbes. ${ }^{46}$

The physical epithelial barrier is reinforced with chemical components, including a glycocalyx formed by mucins bound to the apical surface of the IEC membrane and a sugarrich mucus layer secreted by goblet cells of the intestinal epithelium. This mucus layer is capable of trapping those microbes that lack mucinases, adherence, colonization, or invasion factors and restricting their ability to access the IECs. ${ }^{46}$ Immunochemical members of the intestinal epithelial barrier include microbicidal peptides and proteins, such as defensins, ${ }^{46}$ and the recently identified intestinal angiogenins. Commensal microbes are actually capable of inducing host production of these anti-microbial peptides. Current evidence also suggests that the commensal flora can induce slgA with low affinity but with broad specificity to facilitate the sequestration of gut microbes to the luminal side of the gut epithelium. ${ }^{47}$ The physical epithelial barrier is not absolute, however. Controlled transport of live microbes across the epithelial barrier is critical for the induction of adaptive immune effectors ( $B$ and $T$ cells) with both protective and tolerant immune function.

Lamina propria dentritic cells have been shown to extend dendrites through the intestinal epithelium and into the lumen where they can sample antigen. IgA binding to commensal flora and luminal antigen facilitates adherence of these antigens to the $\mathrm{M}$ cells of Peyer's patches ${ }^{48}$ and regulates transport of antigens across the epithelial barrier by $\mathrm{M}$ cells. ${ }^{49}$

Recently, enterocytes themselves have been shown to phagocytose enteric microbes both in vitro and in vivo, thus mediating controlled translocation of these microbes across the intestinal epithelium..$^{50}$ It was demonstrated that intestinal epithelial cell lines expressing the innate immune receptor TLR4 were capable of phagocytosing the Gram negative bacterium E. coli in a TLR4-dependent fashion. ${ }^{50}$ Such cross talk between antigen-experienced epithelial cells and gut-resident immune effector cells may be critical to the maintenance of immune tolerance to luminal contents.

Commensals that cross the epithelial layer are phagocytosed by macrophages resident in the gastrointestinal mucosa. They are efficiently destroyed by superoxide and inducible nitric oxide generation among other biocidal mechanisms. ${ }^{51,52}$ Moreover, Smythies et al. ${ }^{53}$ have identified a population of human intestinal macrophages that neither express innate immune receptors, such as the lipopolysaccharide receptor CD14, nor produce proinflammatory cytokines, such as IL-1, IL12 , and TNF-a, in response to inflammatory microbial stimuli, in contrast to blood monocytes. Yet, while these intestinal macrophages exhibit 'inflammatory anergy', they are still fully capable of phagocytosis and bacteriocidal activity. ${ }^{53}$ These characteristics make this population of intestinal macrophages ideal subepithelial sentinels. They can destroy luminal microbes with systemic inflammatory potential before these microbes interface with the systemic immune system without eliciting inflammatory changes in the gut that would upset gut homeostasis.

The restriction of commensal-bacteria bearing dendtritic cells to the mesentric lymph nodes can allow robust, local, protective mucosal immune responses to the gut flora (including slgA production) in the absence of unneeded systemic immune responses. mesentric lymph nodes, thus, function as the center of immune privilege in the GALT. ${ }^{54}$

\section{Tregs and tolerance to dietary and commensal antigens}

Immunoquiescence to the gut commensal and dietary antigens is also facilitated by diverse immunoregulatory cells that actively suppress effector immune responses. Several different subsets of Tregs have been implicated in mucosal tolerance induction at the luminal/intestinal epithelial interface.

Intestinal epithelial and stromal-derived mediators promote the generation of immunoregulatory cells in the intestines; tryptophan-catabolizing enzyme indoleamine 2,3-dioxygenase (IDO)-mediated immunoregulation may be particularly relevant in the gut, as IECs constitutively express high levels of IDO. ${ }^{55-57}$ Newberry et al. ${ }^{58}$ demonstrated that non-bone marrow derived stromal cells in the intestinal lamina propria express basal levels of the enzyme cyclooxygenase 2 (COX2) and its arachidonic acid metabolite prostaglandin E2 (PGE2). As both COX2 and 
PGE2 have been shown to prevent inflammatory responses to dietary antigens, this finding implicated intestinal production of COX2 and its arachidonic acid metabolite in the modulation of the immune response to luminal intestinal antigens. ${ }^{58}$ Other work has shown that enterocytes constitutively express thymic stromal lymphopoietin and preferentially induce 'noninflammatory' dendritic cells, which secrete IL-10 and IL-6 and induce Th responses that support the production of barrier protective mucosal IgA. ${ }^{59}$ Smythies et al. ${ }^{53}$ have reported that cellular components of the intestinal stroma, namely lamina propria mast cells, secrete TGF-b capable of driving blood monocytes to take on the phenotype of normal intestinal macrophages, inflammatory anergy, coupled with potent phagocytic and bacteriocidal activity in the face of a plethora of immunostimulatory commensal bacteria.

Immune privilege collapse in the gut:

\section{Inflammatory bowel disease (IBD) and food allergy}

In genetically predisposed individuals, breakdown in gut immune privilege can lead to disease at both ends of the Th1/ Th2 spectrum, as shown in the figure below. ${ }^{20}$

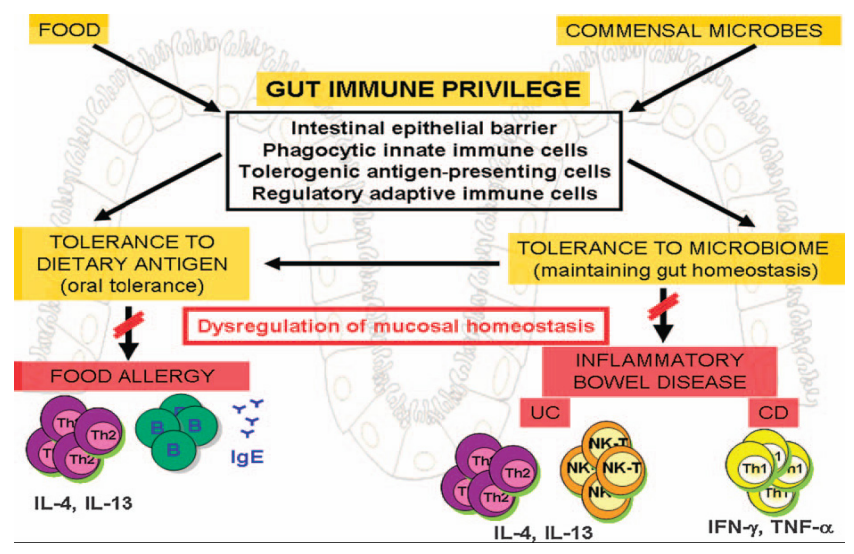

Figure: Immune privilege in the gut consists of tolerance to dietary antigens and to commensal microbes.

Food allergy, an adverse immune response to dietary proteins, affects $6-8 \%$ of children and $2-4 \%$ of adults and is characterized by gastrointestinal and extraintestinal symptoms and the production of large amounts of the Th2-dependent IgE..$^{60}$ Both clinical studies and animal models suggest that alterations in the intestinal microbiome (for example, a reduction in commensal flora or environmental floral burden secondary to increased hygiene, vaccination, and antibiotic use) or in microbiome signaling through TLRs predispose to food allergy. The pathogenesis of food allergy may also be linked to deficiencies in number and function of the $\mathrm{CD} 4^{+} \mathrm{CD} 25^{+} \mathrm{Foxp} 3^{+}$ Treg cell compartment in genetically susceptible patients. ${ }^{61-63}$

While the absence of gut immune privilege to food results in food allergy, the consequences of immune privilege collapse to commensal gut flora is IBD.

IBD comprises mainly ulcerative colitis (UC) and Crohn's disease (CD) characterized by long term intestinal inflammation attributable to an exaggerated immune response. ${ }^{64}$ Earlier studies showed that in healthy humans, tolerance exists toward resident intestinal flora but is broken in active IBD. ${ }^{65}$ This finding was recapitulated in a mouse model of experimental colitis in which health was restored by treatment with the immunoregulatory cytokine IL-10 or antibodies to the proinflammatory cytokine IL-12. ${ }^{66}$ Ulcerative colitis (UC) is believed to encompass a heterogeneous group of diseases with different pathogenic mechanisms but common symptomatology. ${ }^{67}$ Patients with UC experience contiguous inflammation of their colonic LP. ${ }^{67}$ A spontaneously remitting and relapsing disorder, UC falls on the Th2 spectrum of intestinal disorders, as it is characterized by abnormally high amounts of the Th2 cytokines IL- 4 and IL-13 produced by intestinal LP CD4 ${ }^{+}$ T cells and natural killer (NK) T cells. ${ }^{67}$ The pathogenesis of UC is largely unknown, although animal models of UC appear to be driven by dysregulated immune responses to commensal microflora with subsequent generation of cross-reactive autoantibodies. ${ }^{68-72}$ Studies of Abreu et al. ${ }^{73,74}$ have implicated defects in TLR-mediated bacterial antigen sampling in the pathogenesis of the disease. Perhaps, a lack of microbially generated Tregs allows for inappropriate expansion of $\mathrm{CD}^{+}$ Th2 and NK T-cell effector compartments, leading to excessive Th2 cytokine production and disease pathogenesis. ${ }^{67}$

In contrast to UC, Crohn's disease is a Th1-dominant IBD; in patients with Crohn's disease, intestinal homeostasis is perturbed, and the production of inflammatory cytokines such as IFN-g and TNF-a is increased in the lamina propria. ${ }^{75}$ The intestinal tract in Crohn's disease is characterized by patchy transmural inflammation. ${ }^{75}$ Human studies suggest that primary defects in intestinal barrier function include altered mucus layer thickness ${ }^{76,77}$ and epithelial cell gene expression, ${ }^{78}$ leading to increased permeability of the intestinal epithelium. ${ }^{79}$ Increased intestinal permeability may facilitate barrier breach by commensal microbes and probable breakdown in systemic immunological ignorance to the commensal flora, contributing to disease pathogenesis. Lodes et al. ${ }^{80}$ found elevated antiflagellin serum IgG levels in both Crohn's disease patients and mouse models of IBD, supporting the conclusion that bacterial flagellins are the dominant antigen in humans with Crohn's disease. Since TLR5 is only expressed on the basolateral surfaces of IECs, ${ }^{81}$ only microbes that have breached the epithelial layer can signal via TLR5, generating an active immune response to the protein. Defects in immunomodulatory mediator production by the intestinal epithelium of Crohn's disease patients may also contribute to disease pathogenesis. Rimoldi 
et al. reported that the immunomodulatory compound thymic stromal lymphopoietin was undetectable in epithelial cells from Crohn's disease patients and that Crohn's disease epithelial cells cannot induce 'non-inflammatory dendritic cells generated by healthy human colonic epithelial cells. ${ }^{59}$ Defects in the $\mathrm{CD}^{+}$Treg compartment have also been implicated in disease pathogenesis. ${ }^{82}$ Future therapies for IBD may center on modulation of the gut luminal microflora in order to restore this Tregs compartment. To this end, Ince et al. ${ }^{83}$ have shown that colonization with helminth, which downregulates colitis in both human and animal models of IBD. ${ }^{84}$ upregulated the expression of TLR4 on lamina propria T cells, thus promoting the production of lipopolysachharide-induced TGF-b but not proinflammatory cytokines. ${ }^{83}$ In short, the addition of a helminth to an inflammatory intestinal environment may help restore the balance between commensal microbes and host, thus alleviating disease pathology.

\section{CONCLUSION}

The pathogens and harmless antigens from the bacterial flora and food constantly expose the mucosal surface of the gastrointestinal tract. The mucosal epithelial cells act not only as a physical barrier, but also as a local immune system, which plays a vital role in defense and self-tolerance. Dysregulation of this complex immune response can result in a collapse of immune privilege leading into chronic intestinal inflammation. Hence, the knowledge of the homeostatic regulation of the intestinal immune system paves the way for the development of the new immunomodulatory drugs in the therapy of IBD. Moreover, the generation of immune mediated cells through orally fed antigens could be the area of research in the treatment of certain autoimmune diseases.

\section{REFERENCES:}

1) Griebel PJ, Hein WR. Expanding the role of Peyer's patches in B-cell ontogeny. Immunol Today 1996; 17:309.

2) Zarember KA, Godowski PJ. Tissue expression of human Toll-like receptors and differential regulation of Toll-like receptor mRNAs in leukocytes in response to microbes, their products, and cytokines. J Immunol 2002; 168:55461.

3) Kelsall BL. Subset of dendritic cells in the Peyer's patch. In: Stallmach A, Zeitz M, Strober W, MacDonald TT, Lochs $\mathrm{H}$ (eds). Induction and modulation of gastrointestinal inflammation. Kluwer, Dordrecht; 1999, p 24.

4) Leavy O. Antibody responses: Neighbourly help in the gut. Nature Reviews Immunology 2007;7:4.

5) Brandtzaeg P, Farstad IN, Helgeland L. Phenotypes of T cells in the gut. Chem Immunol 1998;71:1-26.

6) Zeitz M, Quinn TC, Graeff AS et al. Mucosal T cells provide helper function but do not proliferate when stimulated by specific antigen in lymphogranuloma venerum proctitis in non-human primates. Gastroenterology 1988;94:353-66.

7) Targan SR, Deem RL, Liu M, et al. Definition of a lamina propria $T$ cell responsive state. Enhanced cytokine responsiveness of $T$ cells stimulated through the $C D 2$ pathway. J Immunol 1995; 154:664-75.

8) Boirivant M, Fuss I, Fiocchi C, et al. Hypoproliferative human lamina propria $T$ cells retain their capacity to secrete lymphokines when stimulated via the $\mathrm{CD} 2 / \mathrm{CD} 28$ accessory signaling pathway. Proc Assoc Am Physiol 1996; 108:55-67.

9) James SP, Kwan WC, Sneller MC. T cells in inductive and effector compartments of the intestinal mucosal immune system of nonhuman primates differ in lymphokine mRNA expression, lymphokine utilization, and regulatory function. J Immunol 144:1251-6.

10) James SP, Fiocchi C, Graeff AS, et al. Phenotypic analysis of lamina propria lymphocytes. Predominance of helperinducer and cytotoxic T-cell phenotypes and deficiency of suppressor-inducer phenotypes in Crohn's disease and control patients. Gastroenterology 1986; 91:1483-9.

11) Schieferdecker $H L$, Ullrich $R$, Weiss-Breckwoldt $A N$, et al. The HML-1 antigen of intestinal lymphocytes is an activation antigen. J Immunol 1990;144:2541-9.

12) Schieferdecker HL, Ullrich $R$, Hirseland $H$, et al. T cell differentiation antigens on lymphocytes in human intestinal lamina propria. J Immunol 1992;149:2816-22.

13) London SD, Rubin DH. Functional role of mucosal cytotoxic lymphocytes. In: Ogra PL, Mestecky J, Lamm $\mathrm{ME}$, et al (eds). Mucosal immunology, vol 1. Academic Press, San Diego; 1999, p 643.

14) Deusch K, Lüling F, Reich $K$, et al. A major fraction of human intraepithelial lymphocytes simultaneously expresses the gamma/delta $T$ cell receptor, the CD8 accessory molecule and preferentially uses the $\mathrm{V}$ delta 1 gene segment. Eur J Immunol 1991;21:1053-9.

15) Lundqvist C, Melgar S, Yeung MMW, et al. Intraepithelial 
lymphocytes, evidence for regional specialization and extrathymic $T$ cell maturation in the human gut epithelium. Int Immunol 1995;7:1473-87.

16) Cepek KL, Shaw SK, Parker CM, et al. Adhesion between epithelial cells and $T$ lymphocytes mediated by $\mathrm{E}$-cadherin and the alpha $\mathrm{E}$ beta7 integrin. Nature 1994;372:190-3.

17) Boismenu R, Havran WL. Modulation of epithelial cell growth by intraepithelial gamma delta T cells. Science 1994;266:1253-5.

18) Hoffmann JC, Peters K, Henschke $S$, et al. Role of $T$ lymphocytes in rat 2: 4,6-trinitrobenzene sulphonic acid (TNBS) induced colitis: increased mortality after gammadelta $T$ cell depletion and no effect of alpha beta T cell depletion. Gut 2001;48:489-95.

19) Mayer L, Posnett DN, Kunkel HG. Human malignant $T$ cells capable of inducing an immunoglobulin class switch. J Exp Med 1985;161:134-44.

20) Iweala OI, Nagler CR. Immune privilege in the gut: the establishment and maintenance of non-responsiveness to dietary antigens and commensal flora. Immunological Reviews 2006;|213:82-100.

21) Weiner HL. Oral tolerance: immune mechanisms and treatment of autoimmune diseases. Immunol Today 1997;18:335-43.

22) Chen $Y$, Inobe JI, Weiner HL. Inductive events in oral tolerance in the TCR transgenic adoptive transfer model. Cell Immunol 1997;178:62-8.

23) Kelsall BL, Ehrhardt RO, Strober W. Peyer's patch dendritic cells: phenotypic characterization by FACS analysis and in vitro primary and secondary $\mathrm{T}$ cell responses using a TCR-transgenic mouse model. J Cell Biochem 1994; Suppl 18D.

24) Marth T, Strober W, Kelsall BL. High dose oral tolerance in ovalbumin TCR-transgeneic mice: systemic neutralization of IL-12 augments TGF-beta secretion and T cell apoptosis. J Immunol 1996;157:2348-57.

25) Chen $Y$, Inobe JI, Marks $P$, et al. Peripheral deletion of antigen-reactive T-cells in oral tolerance. Nature 1995;376:177-80.

26) Finkelmann FD, Lees $A$, Birnbaum $R$, et al. Dendritic cells can present antigen in vivo in a tolerogenic or immunogenic fashion. J Immunol 1996;157:1406-14.

27) Viney JL, Mowat AMI, O'Malley J, et al. Expanding dendritic cells in vivo enhances the induction of oral tolerance. J Immunol 1998;160:5815-25.

28) Dippold W, Wittig B, Schwaeble W, et al. Expression of the intercellular adhesion molecule 1 (ICAM-1, CD54) in colonic epithelial cells. Gut 1993;34:1593-7.

29) Bloom $S$, Simmons $D$, Jewell DP, et al. Adhesion molecules intercellular adhesion molecules-1 (ICAM-1), ICAM-3, and $\mathrm{B} 7$ are not expressed by epithelium in normal and inflamed colon. Clin Exp Immunol 1995;101:157-63.

30) Spahn TW, Fontana A, Faria AMC, et al. Induction of oral tolerance to cellular immune responses in the absence of Peyer's patches. Eur J Immunol 2001;31:1278-87. 31) Akira S, Uematsu S, Takeuchi O. Pathogen recognition and innate immunity. Cell 2006;124:783-801. 32) Iwasaki A, Medzhitov R. Toll-like receptor control of the adaptive immune responses. Nat Immunol 2004;5:987-95.

33) Hacker $H$, Redecke V, Blagoev B, et al. Specificity in Toll-like receptor signalling through distinct effector functions of TRAF3 and TRAF6. Nature 2006;439:204-7. 34) Hoebe K, Janssen EM, Kim SO,et al. Upregulation of costimulatory molecules induced by lipopolysaccharide and double-stranded RNA occurs by Trif-dependent and Trif independent pathways. Nat Immunol 2003;4:12239.

35) Weighardt $H$, Jusek $G$, Mages J, et al. Identification of a TLR4- and TRIF-dependent activation program of dendritic cells. Eur J Immunol 2004;34:558-64. 36) Turnbull EL, Yrlid $U$, Jenkins $C D$, et al. Intestinal dendritic cell subsets: differential effects of systemic TLR4 stimulation on migratory fate and activation in vivo. J Immunol 2005;174:1374-84.

37) Pasare C, Medzhitov R. Control of B-cell responses by Toll-like receptors. Nature 2005;438:364-8. 38) Pasare C, Medzhitov R. Toll pathway-dependent blockade of CD4+CD25+ T cell-mediated suppression by dendritic cells. Science 2003;299:1033-6.

39) Sutmuller RP, den Brok MH, Kramer $M$, et al. Toll-like receptor 2 controls expansion and function of regulatory T cells. J Clin Invest 2006;116:485-94.

40) Blander JM, Medzhitov R. Toll-dependent selection of microbial antigens for presentation by dendritic cells. Nature 2006;440:808-12.

41) Scupham AJ, Presley LL, Wei B, et al. Abundant and diverse fungal microbiota in the murine intestine. Appl 
Environ Microbiol 2006;72:793-801.

42) Macfarlane S, Woodmansey EJ, Macfarlane GT. Colonization of mucin by human intestinal bacteria and establishment of biofilm communities in a two-stage continuous culture system. Appl Environ Microbiol 2005;71:7483-92.

43) Neish AS, Gewirtz AT, Zeng $H$, et al. Prokaryotic regulation of epithelial responses by inhibition of IkappaB-alpha ubiquitination. Science 2000;289:1560-3.

44) Collier-Hyams LS, Sloane V, Batten BC, et al. Cutting edge: bacterial modulation of epithelial signaling via changes in neddylation of cullin-1. J Immunol 2005;175:4194-8.

45) Kelly D, Campbell Jl, King TP, et al. Commensal anaerobic gut bacteria attenuate inflammation by regulating nuclear-cytoplasmic shuttling of PPAR-gamma and RelA. Nat Immunol 2004;5:104-112.

46) Sansonetti PJ. War and peace at mucosal surfaces. Nat Rev Immunol 2004;4:953-64.

47) Macpherson AJ, Geuking MB, McCoy KD. Immune responses that adapt the intestinal mucosa to commensal intestinal bacteria. Immunology 2005;115:153-62. 48) Mantis NJ, Cheung MC, Chintalacharuvu KR, et al. Selective adherence of IgA to murine Peyer's patch $M$ cells: evidence for a novel IgA receptor. J Immunol 2002;169:1844-51.

49) Weltzin R, Lucia-Jandris $P$, Michetti $P$, et al. Binding and transepithelial transport of immunoglobulins by intestinal $M$ cells: demonstration using monoclonal IgA antibodies against enteric viral proteins. J Cell Biol 1989;108:1673-85.

50) Neal MD, Leaphart C, Levy R, et al. Enterocyte TLR4 mediates phagocytosis and translocation of bacteria across the intestinal barrier. J Immunol 2006;176:30703079.

51) Shiloh MU, MacMicking JD, Nicholson S, et al. Phenotype of mice and macrophages deficient in both phagocyte oxidase and inducible nitric oxide synthase. Immunity 1999;10:29-38.

52) Sansonetti P. Phagocytosis of bacterial pathogens: implications in the host response. Semin Immunol 2001;13:381-90.

53) Smythies LE, Sellers $M$, Clements RH, et al. Human intestinal macrophages display profound inflammatory anergy despite avid phagocytic and bacteriocidal activity. J Clin Invest 2005;115:66-75.
54) Macpherson AJ, Smith K. Mesenteric lymph nodes at the center of immune anatomy. J Exp Med 2006;203:497500 .

55) Cook JS, Pogson $\mathrm{Cl}$, Smith $\mathrm{SA}$. Indoleamine 2,3-dioxygenase. A new, rapid, sensitive radiometric assay and its application to the study of the enzyme in rat tissues. Biochem J 1980;189:461-6.

56) Takikawa O, Yoshida R, Kido R, et al. Tryptophan degradation in mice initiated by indoleamine 2,3-dioxygenase. J Biol Chem 1986;261:3648-53.

57) Gurtner GJ, Newberry RD, Schloemann SR, et al. Inhibition of indoleamine 2,3-dioxygenase augments trinitrobenzene sulfonic acid colitis in mice. Gastroenterology 2003;125:1762-73.

58) Newberry RD, McDonough JS, Stenson WF, et al. Spontaneous and continuous cyclooxygenase-2dependent prostaglandin E2 production by stromal cells in the murine small intestine lamina propria: directing the tone of the intestinal immune response. J Immunol 2001;166:4465-72.

59) Rimoldi M, Chieppa M, Salucci V,et al. Intestinal immune homeostasis is regulated by the crosstalk between epithelial cells and dendritic cells. Nat Immunol 2005;6:507-14.

60) Sicherer SH, Sampson HA. Food allergy. J Allergy Clin Immunol 2006;117(Suppl.):S470-5.

61) Hori S, Nomura T, Sakaguchi S. Control of regulatory $T$ cell development by the transcription factor Foxp3. Science 2003;299:1057-61.

62) Karlsson MR, Rugtveit J, Brandtzaeg P. Allergenresponsive $\mathrm{CD} 4+\mathrm{CD} 25+$ regulatory $\mathrm{T}$ cells in children who have outgrown cow's milk allergy. J Exp Med 2004;199:1679-88.

63) Ling EM, Smith $T$, Nguyen $X D$, et al. Relation of CD4+CD25+ regulatory T-cell suppression of allergen driven T-cell activation to atopic status and expression of allergic disease. Lancet 2004;363:608-15.

64) Xia B, Crusius J, Meuwissen S, et al. Inflammatory bowel disease: definition, epidemiology, etiologic aspects, and immunogenetic studies. World J Gastroenterol 1998;4:446-58.

65) Duchmann R, Kaiser I, Hermann E, et al. Tolerance exists towards resident intestinal flora but is broken in active inflammatory bowel disease (IBD). Clin Exp Immunol 1995;102:448-55. 
66) Duchmann R, Schmitt E, Knolle $P$, et al. Tolerance towards resident intestinal flora in mice is abrogated in experimental colitis and restored by treatment with interleukin-10 or antibodies to interleukin-12. Eur J Immunol 1996;26:934-8.

67) Targan SR, Karp LC. Defects in mucosal immunity leading to ulcerative colitis. Immunol Rev 2005;206:296-305.

68) Mizoguchi E, Mizoguchi A, Chiba C, et al. Antineutrophil cytoplasmic antibodies in T-cell receptor alphadeficient mice with chronic colitis. Gastroenterology 1997;113:1828-35

69) Mizoguchi A, Mizoguchi E, Chiba C, et al. Role of appendix in the development of inflammatory bowel disease in TCR-alpha mutant mice. J Exp Med 1996;184:707-15.

70) Mizoguchi A, Mizoguchi E, Chiba C, et al. Cytokine imbalance and autoantibody production in $\mathrm{T}$ cell receptor-alpha mutant mice with inflammatory bowel disease. J Exp Med 1996;183:847-56.

71) Heller F, Fuss IJ, Nieuwenhuis EE, et al. Oxazolone colitis, a Th2 colitis model resembling ulcerative colitis, is mediated by IL-13-producing NK-T cells. Immunity 2002;17:629-38.

72) Iqbal $N$, Oliver JR, Wagner $F H$, et al. T helper 1 and $T$ helper 2 cells are pathogenic in an antigen-specific model of colitis. J Exp Med 2002;195:71-84.

73) Abreu MT, Arnold ET, Thomas LS, et al. TLR4 and MD-2 expression is regulated by immune-mediated signals in human intestinal epithelial cells. J Biol Chem 2002;277:20431-7.

74) Abreu MT, Vora P, Faure E, et al. Decreased expression of Toll-like receptor- 4 and MD-2 correlates with intestinal epithelial cell protection against dysregulated proinflammatory gene expression in response to bacterial lipopolysaccharide. J Immunol 2001;167:1609-
16.

75) Cobrin GM, Abreu MT. Defects in mucosal immunity leading to Crohn's disease. Immunol Rev 2005;206:27795.

76) Pullan RD. Colonic mucus, smoking and ulcerative colitis. Ann R Coll Surg Engl

1996;78:85-91.

77) Pullan RD, Thomas GA, Rhodes $M$, et al. Thickness of adherent mucus gel on colonic mucosa in humans and its relevance to colitis. Gut 1994;35:353-9.

78) Fukushima K, Yonezawa H, Fiocchi C. Inflammatory bowel disease-associated gene expression in intestinal epithelial cells by differential cDNA screening and mRNA display. Inflamm Bowel Dis 2003;9:290-301.

79) Hollander D. Intestinal permeability, leaky gut, and intestinal disorders. Curr Gastroenterol Rep 1999;1:4106.

80) Lodes MJ, Cong Y, Elson CO, et al. Bacterial flagellin is a dominant antigen in Crohn disease. J Clin Invest 2004;113:1296-306.

81) Gewirtz AT, Navas TA, Lyons $S$, et al. Cutting edge: bacterial flagellin activates basolaterally expressed TLR5 to induce epithelial proinflammatory gene expression. J Immunol 2001;167:1882-5.

82) Brimnes J, Allez M, Dotan I, et al. Defects in CD8+ regulatory $T$ cells in the lamina propria of patients with inflammatory bowel disease. J Immunol 2005;174:581422.

83) Ince $M N$, Elliott $D E$, Setiawan $T$, et al. Cutting edge: Heligmosomoides polygyrus induces TLR4 on murine mucosal $T$ cells that produce TGFbeta after lipopolysaccharide stimulation. J Immunol 2006;176:726-9. 